\title{
Long-Time Decay of Velocity Autocorrelation Function of Two-Dimensional Lattice Gas Cellular Automata
}

\section{Frenkel}

FOM Institute for Atomic and Molecular Physics, P.O.Box 41883, NL-1009 DB Amsterdam, The Netherlands

\begin{abstract}
A method is introduced to compute the velocity autocorrelation function (VACF) of a tagged particle in a lattice gas. This method yields at least a million-fold improvement over the conventional method to measure such correlation functions in lattice-gas cellular automata (LGCA's). For lattice Lorentz gases, the gain is even several orders of magnitude larger. Using this method, a $t^{-1}$-algebraic tail in the VACF of a tagged particle in a two-dimensional LGCA is clearly observed.
\end{abstract}

\section{Introduction}

One of the attractive features of lattice gas models is that they are ideally suited to serve as a testing ground for concepts in kinetic theory. The reason is twofold: on the one hand the very simple structure of most lattice-gas models often makes it possible to work out in closed form the consequences of a particular approximation scheme in kinetic theory. On the other hand, lattice-gas models are ideally suited for computer simulation. Thus approximate schemes in kinetic theory can be tried out on lattice gas models before applying them to more realistic models of fluids or solids. In this paper I describe the computational scheme that makes it possible to compute certain transport properties of lattice gases with hitherto unachievable accuracy.

This method is then applied to the calculation of long-time tails in the tagged-particle velocity ACF in two-dimensional lattice-gas cellular automata. Such calculations are of fundamental interest for the following reason: The discovery of algebraic long-time tails in the velocity ACF of a tagged particle was first reported in a classic paper by Alder and Wainwright [1]. Such longtime tails are the consequence of coupling between particle diffusion and shear modes in the fluid. To a first approximation the leading term in the long-time tail of the velocity ACF is

$$
\left\langle v_{x}(0) v_{x}(t)\right\rangle \approx \frac{D-1}{D} \frac{1}{\rho\left(4 \pi\left(D_{0}+\nu_{0}\right) t\right)^{D / 2}} \equiv \frac{d_{0}}{t^{D / 2}},
$$

where $\rho$ is the number density, $D_{0}$ the 'bare' self-diffusion constant, $\nu_{0}$ the 
kinematic viscosity and $D$ the dimensionality. Ever since the discovery of these hydrodynamic tails, it has been realized that a consistent description of modecoupling effects in a two-dimensional fluid would result in a long-time tail that decays faster than $t^{-1}$, because in $2 D$ the self-diffusion constant itself diverges (see e.g. [2]). In fact, de Schepper and Ernst [3] computed the coefficient $\left(d_{1}\right.$ in eqn. (2)) of the first correction to the $t^{-1}$ tail for a system of hard disks. They predicted that this correction should be negative and proportional to $\log \left(t / t_{0}\right) / t$ (where $t_{0}^{-1}$ is the initial decay rate of the velocity ACF):

$$
\left\langle v_{x}(0) v_{x}(t)\right\rangle \approx d_{0} / t+d_{1} \log \left(t / t_{0}\right) / t+\ldots \text { for } t / t_{0} \gg 1
$$

Forster et al. [4] argued that as $t \rightarrow \infty$, the tail should be renormalized to $1 /(t \sqrt{\log t})$.

Thus far it has not been possible to compare these predictions directly with computer simulation data. The reason is that such a comparison requires accurate knowledge of the velocity ACF for very long times (many tens to hundreds of collision times). This requires very long simulations on very large systems (to avoid problems due to spurious correlations caused carried by sound waves in periodic systems).

Using the techniques presented in the present paper it is possible to compute tagged particle velocity ACF's in a simple model for a hydrodynamic fluid, namely a two-dimensional lattice-gas cellular automaton. These correlation functions can be followed over hundreds of collision times. This opens the way to carry out an estimate of the magnitude of corrections to the $t^{-1}$ long-time tail.

In order to explain the numerical scheme, I shall first describe its application to lattice Lorentz gases. Thereafter I indicate how the method can be extended to study long-time tails in the velocity autocorrelation function of a tagged particle in a lattice gas cellular automaton. It turns out that, depending on the application, the present method requires a factor $10^{7}-10^{10}$ less computer time than the more 'brute-force' methods that have been applied thus far.

Let us first consider a lattice-Lorentz gas with 'bond' disorder. This is a model system where a random walker performs a hopping motion along the nearest-neighbor bonds of a square (2D) or simple-cubic (3D) lattice. The moving particle can jump with equal probability along any of the 4 (or 6 ) bonds to a neighboring site. However, if the bond is 'broken', the particle remains at its original site until the next trial move. In the bond-disorder model, a finite fraction of the bonds is broken. The broken bonds are distributed randomly over the lattice. Two equivalent versions of this random walk process can be formulated: a) a discrete time version in which a new trial move is attempted every unit time and b) a continuous time version where the waiting times between successive trial moves are Poisson distributed. It is clear that statistical properties of the continuous-time model, such as time correlation function $C(t)$, can be 
obtained as a weighted average over all n-step correlations of the corresponding discrete-time model $C_{D}(n)$ :

$$
C(t)=\frac{1}{2} C_{D}(0) \delta\left(0_{+}\right)+\sum_{n=1}^{\infty} C_{D}(n) \frac{t^{n}}{n !} \exp (-t)
$$

assuming an average rate of one jump per unit time for the continuous time model. It turns out that the theory for transport in lattice Lorentz gases is formulated most compactly for the continuous-time model, whereas the simulations are performed most conveniently for the discrete-time version.

Let us now consider the numerical computation of the velocity correlation function of a lattice-Lorentz gas. The discrete nature of the model leads to the following natural definitions of the 'velocity': the velocity $v_{\alpha}\left(t_{n}\right)$ of a random walker at discrete time $t_{n}$ in direction $\alpha$ is $+1(-1)$ if the random walker performed a jump in the $+\alpha(-\alpha)$ direction in time interval $\mathrm{n}$, and is 0 otherwise. With this definition it is easy to see how to compute the velocity auto-correlation function $(\mathrm{ACF}) \phi(t) \equiv\left\langle\mathbf{v}(0) . \mathbf{v}\left(t_{n}\right)\right\rangle$. In principle this quantity can be computed by considering all possible starting positions of the random walker at time $t=0$ and then averaging over all possible random walks that this particle can perform in a time interval $t_{n}$. The problem with this 'brute force' approach is that very many random walks must be sampled to obtain a reasonable statistical accuracy. To give a specific example: for a 2D lattice-Lorentz gas with a low concentration of broken bonds (say $p=0.01$, the long time tail in the velocity ACF is expected to decay as $\phi(t) \approx p / t^{2}$. Hence, if we are interested in the long-time behavior (e.g. $t=100)$, the amplitude of $\phi(t)$ will be $O\left(10^{-6}\right)$. In order to obtain a signal-to-noise ratio of $O(1)$ we should therefore sample over $O\left(10^{12}\right)$ independent random walks. Using an efficient random walk procedure [5] such a simulation would require some $10^{2}$ hours of CPU time on a Cyber 205 vector computer.

In fact, a method has been developed to reduce the required computer time by a factor $10^{2}[6]$, but this technique will not work for lattice-gas cellular automata, to be considered below. Anyway, the approach described in the present paper results in far greater computational gains.

Let us consider the random walk problem that we wish to solve in some detail. In order to compute $\langle\mathbf{v}(0) . \mathbf{v}(t)\rangle$ we could pick a site $\mathbf{X}$ on the lattice at $t=1 / 2$ (in order to minimize confusion we compute velocities at integer times and positions at half integer times) pick a point $\mathbf{X}^{\prime}$ at time $t=t_{n}-\frac{1}{2}$ that can be reached in a possible random walk in time $t_{n}-1$, compute the product $\mathbf{v}(0) \cdot \mathbf{v}\left(t_{n}\right)$ and average over: 1$)$ all possible random walks linking $\mathbf{X}\left(t=\frac{1}{2}\right)$ and $\left.\mathbf{X}^{\prime}\left(t=t_{n}-\frac{1}{2}\right), 2\right)$ all $\mathbf{X}^{\prime}$ and 3$)$ all $\mathbf{X}$.

First, consider the computation of the velocity at $t_{n}$. If the random walker is at site $\mathbf{X}^{\prime}$ at time $t_{n}-\frac{1}{2}$ then it can jump to any of the neighboring sites with equal probability, unless any of the bonds connected to $\mathbf{X}^{\prime}$ is broken. 
Let us first assume that all bonds connected to $\mathbf{X}^{\prime}$ are open. Then clearly the average velocity of the random walker in the time interval centered at $t_{n}$ is 0 . Only if one or more bonds are broken can the random walker have a non-zero average velocity in the interval $\left[t_{n}-\frac{1}{2}, t_{n}+\frac{1}{2}\right]$. Let us denote this velocity by $\mathbf{v}\left(\mathbf{X}^{\prime}\right)$. Using this definition, we note that the average velocity of a random walker at time $t=0$ (i.e. just before its arrival at $\mathbf{X}$ is equal to $-\mathbf{v}(\mathbf{X})$. Clearly, if there are no broken bonds around $\mathbf{X}, \mathbf{v}(\mathbf{X})=0$ and all random walks starting at $\mathbf{X}$ do not contribute to $\langle\mathbf{v}(0) \cdot \mathbf{v}(t)\rangle$. From now on we restrict our attention to those random walks for which $\mathbf{v}(\mathbf{X}) \neq 0$. The actual number of lattice sites for which $\mathbf{v}(\mathbf{X}) \neq 0$ can easily be counted once the distribution of broken bonds has been specified. The average fraction of sites for which $\mathbf{v}_{\alpha}(\mathbf{X}) \neq 0$. for any direction $\alpha$ is equal to $2 p(1-p)$, where $p$ is the fraction of broken bonds.

Now let us consider the computation of $\langle\mathbf{v}(0) \cdot \mathbf{v}(t)\rangle$. For $t=0$ the answer is simply $(1-p)$. For $t=1$, we obtain $-\overline{v^{2}(X)}$ where the bar denotes averaging over all lattice sites. In order to compute $\langle\mathbf{v}(0) . \mathbf{v}(t)\rangle$ for $t>1$, we note that if at time $t-\frac{1}{2}$ a particle is at position $\mathbf{X}$ then after one more time-step, it has a probability $1 / 2 D(D=$ dimensionality) to be on any of the $\ell(\leq 2 D)$ connected nearest neighbor sites of $\mathbf{X}$ and a probability $(2 D-\ell) / 2 D$ to be still at site $\mathbf{X}$. The average velocity of this random walker at time $t+1$ is therefore

$$
\mathbf{v}(t+1)=\sum_{\text {all } \mathbf{X}^{\prime} \text { connected to } \mathbf{X}} \frac{1}{2 D} \mathbf{v}\left(\mathbf{X}^{\prime}\right)
$$

where the original site $\mathbf{X}$ is counted as 'connected' to itself for every broken bond present. We now attribute this average velocity $\mathbf{v}(t+1)$ to the site $\mathbf{X}$ and denote it by $\mathbf{v}^{(\mathbf{2})}(\mathbf{X})$. Clearly, $\langle\mathbf{v}(0) \cdot \mathbf{v}(2)\rangle=-\overline{\mathbf{v}(\mathbf{X}) \cdot \mathbf{v}^{(\mathbf{2})}(\mathbf{X})}$. Analogously, by iteration we obtain $\mathbf{v}^{(\mathbf{3})}(\mathbf{X})$ from $\mathbf{v}^{(\mathbf{2})}(\mathbf{X})$, and so on.

Note that in this way we compute the contribution to $\langle\mathbf{v}(0) . \mathbf{v}(t)\rangle$ from all possible random walks. Hence, once we have specified the distribution of broken bonds on the lattice, the present computation of $\langle\mathbf{v}(0) . \mathbf{v}(t)\rangle$ is exact. Statistical errors are only due to the fact that the velocity correlation function should be computed for a large number of independently generated realizations of the bond disorder. The other point to note is that the iteration scheme requires only very simple matrix operations that are eminently vectorizable. To demonstrate the power of the present method, figure 1 shows the long-time tail of the velocity $\mathrm{ACF}$ of a $2 D$ lattice-Lorentz gas with bond disorder.

Note that the statistical error (points) in fig. 1 decreases with increasing time. For the longest times studied in this simulation the statistical error is less than 1 in $10^{10}$. The calculation required 13 minutes of CPU-time on a CYBER 205 vector computer. To achieve the same accuracy by straightforward Monte Carlo sampling would require over $10^{5}$ years of CPU time on the same machine. The difference between the statistical error obtained by the present method and those obtained using the Monte Carlo approach [6] is particularly striking for 


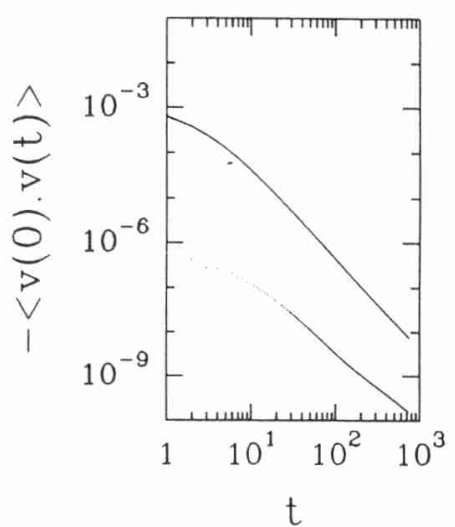

Fig. 1: Velocity autocorrelation function of a random walker on a twodimensional square lattice-Lorentz gas with a fraction of $1 \%$ broken bonds. The drawn curve is the velocity correlation function. The dots represent the statistical error.

long times. Direct sampling over trajectories yields a statistical error that is essentially independent of time. Hence to compute a $t^{-2}$ long-time tail over a time interval that is 10 times longer required a factor $10^{4}$ (!) more computing time $\left(10^{5}\right.$ in $\left.3 \mathrm{D}\right)$. In contrast, the error in the present matrix-method decreases with time. Hence, to study a 10 -times longer correlation interval simple requires a run that is 10 times longer. The relative merit of the present method becomes greater at longer times. In our case the gain with respect to the random walk method was a factor $10^{10}$, but the comparison could have been made even more biased by going to longer times. The comparison of the numerical results for lattice-Lorentz gases will be the subject of a separate paper [7]. In the present paper I shall show how, by a slight extension, the present method can be applied to compute the long-time behavior of velocity ACF's in lattice-gas cellular automata (LGCA's).

At first sight, the computation of the velocity ACF for a 'particle' in a system of lattice-gas CA's seems very different from the corresponding problem of a random walker in a lattice- Lorentz gas. After all, lattice gas cellular automata are meant to model systems of moving, interacting particles. In particular, LGCA's have been constructed as a highly simplified model for an atomic fluid. In the present paper I shall focus on the properties of two-dimensional LGCA's on a $2 D$ triangular lattice (FHP- model [8],[9]), and I shall briefly touch on similar calculations for the square-lattice 'HPP'-model [10]. The techniques can easily be applied to other lattice gases, including 3D models. In LGCA's, the particles are constrained to move along the bonds joining the lattice sites. No two particles can move along the same bond in the same direction. The state of the lattice is completely specified by indicating which links are occupied and which are empty. This implies that lattice-gas particles are indistinguishable. 
The time evolution of the system is governed by the following rules:

1. Propagation: all particles move in one time step (for convenience we choose $\Delta t=1$ ) from their initial lattice position(say $\mathbf{X}$ ) to a new position $\left(\mathbf{X}^{\prime}=\mathbf{X}+\mathbf{c}_{\alpha}\right.$; where $\mathbf{c}_{\alpha}$ is the velocity of species $\left.\alpha\right)$. The velocities $\mathbf{c}_{\alpha}$ are such that at the end of the propagation steps all particles are once more positioned at lattice sites.

2. Collision: the particles at all sites on the lattice undergo a collision that conserves the total number of particles and the total momentum at each site. Usually, these local collision rules are deterministic.

Provided that the lattice has a sufficiently high symmetry (e.g. triangular in 2 dimensions) and the collision rules are sufficiently isotropic (for a discussion, see [11]), it can be shown that the equation that governs the time evolution of the distribution function of such a lattice gas becomes equivalent to the NavierStokes equation for an incompressible fluid in the limit that the flow velocity is much less than the particle velocity, and all spatial variations in the system occur on a scale that is large compared to the mean free path of the lattice gas particles. In this respect LGCA's model atomic fluids.

When attempting to compute the velocity correlation function of a particle in a lattice gas CA, one is immediately confronted with a conceptual problem. As all lattice gas particles are indistinguishable, the velocity correlation function of 'a particle' is ill defined. As soon as a particle has collided it is no longer possible to identify any of the outgoing particles as the original particles whose velocity $\mathrm{ACF}$ we are attempting to compute. To avoid this problem, the particle under consideration must be labeled differently from the rest (say, a 'blue' particle in a sea of 'red' particles). Once the collision rules for all particles have been specified we can then compute the velocity ACF of a single tagged particle. This approach has been pursued by Boon and Noullez for the FHP model [12] and by Binder and d'Humières for the HPP model [13]. The drawback of this approach is that it yields very poor statistics (only 1 tagged particle is permissible) and, with the currently available computer hardware, long-time tails could not be detected.

An alternative approach that effectively side-steps the problem referred to above has been followed by Colvin, Ladd and Alder [14]. These authors compute not the velocity ACF of a tagged particle but the autocorrelation function of the fluid velocity at a lattice site. This method yields somewhat better statistics and made it possible to observe a long-time tail in the site-velocity ACF. However, the site-velocity ACF does not contain the same mode-coupling contributions as the tagged-particle velocity $\mathrm{ACF}$ and is therefore, from a theoretical point of view, of less interest.

Fortunately, the matrix method described in the previous section can be extended in such a way that it becomes possible the compute the velocity ACF of a tagged particle in a lattice-gas cellular automaton with high accuracy. In order to do so we have to impose one restriction on the rules of the lattice gas 

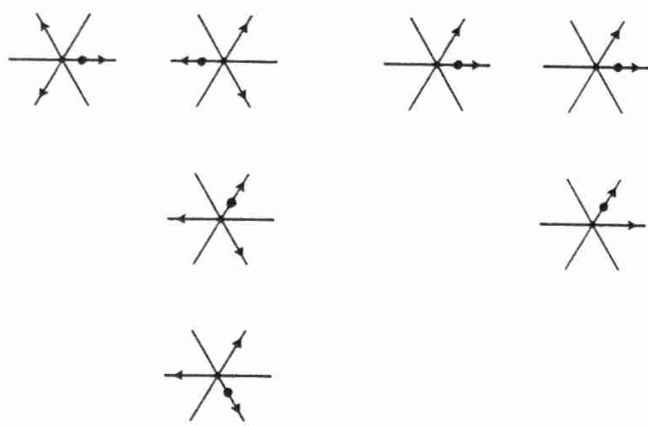

Fig. 2: Left: a 3 body collision that changes the momentum states of the collision partners. Right: a 'collision' that has the same input and output state. The tagged particle is distinguished from the untagged particles by a dot on the arrow.

automaton, namely that the collision rules for a tagged particle with untagged particles result in the occupation of the same output states as in the case of collisions between untagged particles. And, most importantly, the tagged particle has equal probability to be in any of the occupied output states. Hence, for the tagged particle the collision rules are stochastic, although for a 'color-blind' observer, the rules are deterministic. Note also that even in 'collisions' that have the same input and output states (see fig. 2 the tagged particle may still 'collide', i.e. it may change its velocity state.

With these rules, it is obvious that the average velocity of a tagged particle after a collision at site $\mathbf{X}$, depends only on the (colorless) state at that site. In particular, it depends in no way on where the tagged particle was coming from. We can thus define for every (non-empty) site of the lattice the average post collisional velocity that a tagged particle at that site would have:

$$
\mathbf{v}_{\mathbf{X}}(t)=\frac{1}{N_{o c c}(\mathbf{X})} \sum_{\alpha=1}^{N_{o c c}(\mathbf{X})} \mathbf{c}_{\alpha}
$$

where $N_{o c c}(\mathbf{X})$ is the total number of particles at site $\mathbf{X}$ and $\mathbf{c}_{\alpha}$ are the velocities corresponding to the occupied links. At first sight, eqn.(5) may look similar to the expression for the site velocity in a Lorentz gas. The important difference is that for a Lorentz gas the site velocity is fixed by the (time-independent) distribution of random bonds. In contrast, for the LGCA model the site-velocity for a tagged particle changes with every time step. However, apart from this minor modification, we can use basically the same techniques that worked for the Lorentz gases, to compute the velocity $\mathrm{ACF}$ of a tagged particle in a lattice gas cellular automaton.

Consider a tagged particle that is moving at site $\mathbf{X}$ at time $t=0$, moving with a velocity $\mathbf{c}_{\alpha}$. At time $t=1$, the tagged particle will have collided at site $\mathbf{X}+\mathbf{c}_{\alpha}$ and its average post collisional velocity will be $\mathbf{v}_{\mathbf{X}+\mathbf{c}_{\alpha}}(t=1)$. Clearly, 


$$
\langle\mathbf{v}(0) \cdot \mathbf{v}(1)\rangle=\frac{1}{N_{t o t}} \sum_{\alpha, \mathbf{X}} s_{\alpha}(\mathbf{X}, t=0) \mathbf{c}_{\alpha} \cdot \mathbf{v}_{\mathbf{X}+\mathbf{c}_{\alpha}}(t=1)
$$

where $s_{\alpha}(\mathbf{X}, t=0)=1(0)$ if link $\alpha$ at site $\mathbf{X}$ is occupied (empty) at $t=0$.

To compute $\langle\mathbf{v}(0) \cdot \mathbf{v}(2)\rangle$, we simply propagate the tagged particle that is at site $\mathbf{X}+\mathbf{c}_{\alpha}$ to all sites that will be reached from this site in the next time step. For all these sites (denoted by $\{\mathbf{X}(t=2)\}$ we compute the average post collisional velocity, average it over the set $\{\mathbf{X}(t=2)\}$ and this yields the average velocity of the tagged particle after two time-steps.

For the actual implementation of this technique the recipe is then the following:

1. compute the average post collisional velocity of the tagged particle at site $\mathbf{X}$, at time $-t$, for all possible $\mathbf{X}$ on the lattice;

2. propagate this average to all sites that will be reached in one cycle from site $\mathbf{X}$;

3 . for every site thus reached, compute the average of all the averages that have been propagated to it;

4. iterate steps 2 and 3 until $t$ iterations have been made in total.

Note that in order to compute the velocity correlation function of a tagged particle, use was made of all possible starting positions and trajectories that such a particle could have, compatible with the (deterministic) dynamics of the underlying 'uncolored' lattice gas. The only additional averaging is over all possible time origins and over independent initial conditions. The latter averaging would not be necessary if the lattice gas cellular automaton were strictly ergodic. But as the models studied in this paper are known to have spurious invariants [15], averaging over a number of independent initial conditions is advisable.

\section{Results}

Simulations of tagged particle diffusion were carried out for a two-dimensional lattice gas model ('FHP-III', 6 speed-1 particles, one rest-particle, defined in ref. [9]). Also included is one result for the square-lattice HPP model. System sizes of up to $500 \times 500$ lattice points were studied at densities varying from $5 \%$ to $75 \%$ occupancy. The simulations were either performed on a CYBER 205 vector-computer or, for the larger systems, on a NEC-SX2 super-computer.

Figure 3 shows the velocity autocorrelation function of a tagged particle in the $2 \mathrm{D}$ lattice gas at a density $d=0.75$ (i.e. $75 \%$ of all states occupied). The velocity $\mathrm{ACF}$ has been normalized to 1 at $t=0$. Here, and in all other cases shown, correlations were only computed for time intervals less than the shortest time in which any particle could cross the periodic box. 


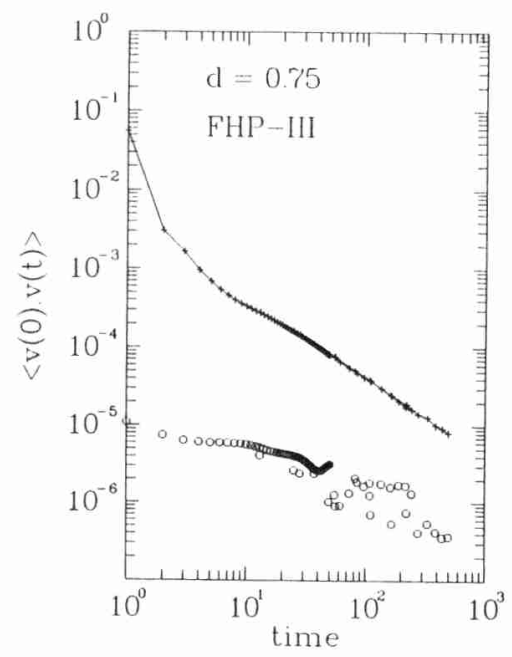

Fig. 3: Example of the normalized velocity autocorrelation function of a tagged particle in a 2D lattice-gas cellular automaton (FHP-III) at a density of $75 \%$. After an initial rapid decay (and overshoot), the velocity ACF approaches a power-law decay with an exponent -1 .

Initially the decay of the velocity ACF is approximately exponential. There is a surprisingly large time-interval where the decay is no longer exponential but not yet algebraic. However, after some 30 collision times the decay appears to become algebraic and is found to follow a $t^{-1}$ decay. This in itself is maybe not surprising, but it is comforting as it has been argued that the hydrodynamic long-time tails observed in computer simulation on continuous systems may be due to a propagation of numerical errors [16]. In the present simulation the discrete dynamics of the lattice gas is solved exactly, hence propagation of numerical errors is ruled out as a factor affecting either the power-law tails or, for that matter, any corrections to the latter.

A quantitative comparison between the measured long-time tails and the predictions of mode-coupling theory is presented in ref. [17]. Here I focus on the general features of the tagged-particle VACF's in lattice gases. Figure 4 shows the behavior of the long-time tail of a lattice gas at a density $d=0.5$. In order to emphasize the long-time behavior, the tail has been multiplied by $t$. As can be seen in the figure, there is a time regime of several dozen collision times where the tail is not yet algebraic even though $t \gg t_{0}$. Of course, the $t_{1}$ tail is only the leading term in the long-time expansion of the VACF. It is conceivable that what is observed is simply the decay of terms that fall off with a higher power of $1 / t$. At this stage there are no theoretical predictions for the intermediate-time behavior of the tagged-particle VACF.

Figure 5 shows the velocity ACF of a tagged particle in the HPP lattice. Clearly, this correlation function also has a long-time tail that decays as $t^{-1}$. However, because of its low symmetry, the HPP model is less useful as a sim- 


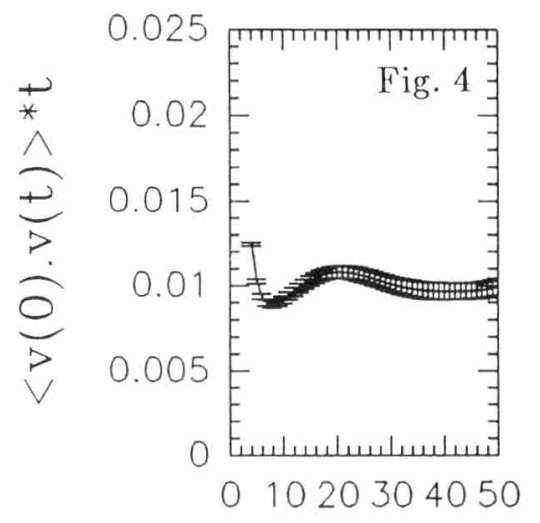

t

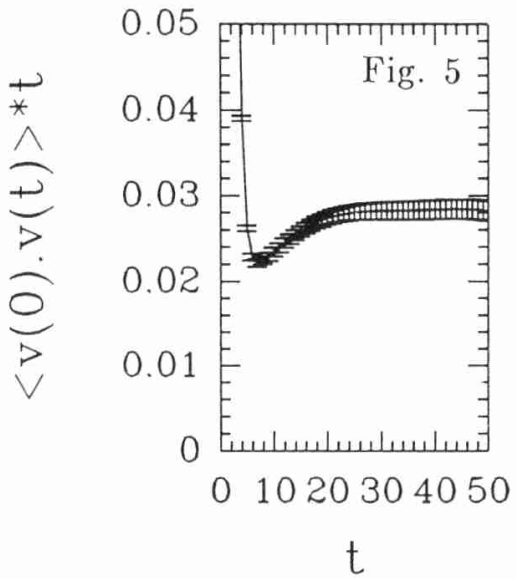

Fig. 4: $t\langle\mathbf{v}(0) \cdot \mathbf{v}(t)\rangle$ for a tagged particle in a 2D lattice-gas cellular automaton (FHP-III) at a density of $50 \%$. If the velocity ACF decays as $t^{-1}$, the function shown in this figure should be a constant. At a density of $50 \%$ the 'collision' time, i.e. the Enskog decay time of the velocity ACF, is $t_{0}=0.553$. Note the non-monotonic approach to the long-time $t^{-1}$ behavior.

Fig. 5: $t\langle\mathbf{v}(0) \cdot \mathbf{v}(t)\rangle$ for a tagged particle in a 2D square lattice-gas cellular automaton (HPP) at a density of $50 \%$. At this density the 'collision' time, i.e. Enskog decay time of the velocity ACF, is $t_{0}=0.811$. After some 30 collision times the velocity ACF decays as $t^{-1}$ within the statistical error.

plified model of a $2 D$ hydrodynamic liquid. For this reason I have not pursued the study of this particular lattice gas.

\section{Conclusions}

I have presented a numerical technique that can be used to compute timecorrelation functions of single-particle properties of tagged particles in lattice gases. The method is very much more efficient than the direct simulation methods that compute correlation functions by averaging over many trajectories. The gain varies from $10^{10}$ for lattice Lorentz gases to $10^{6}$ for LGCA's. Although I have only discussed one very specific application of the technique, the present approach is quite flexible and makes it possible to study many other interesting properties of lattice gas models for transport in solids and liquids.

\section{Acknowledgments}

The work of the FOM Institute is part of the scientific program of FOM and is supported by the 'Nederlandse Organisatie voor Wetenschappelijk Onderzoek' 
(NWO). Computer time on the Amsterdam CYBER 205 and the NEC-SX2 at NLR were made available through a grant by the NFS (Nationaal Fonds Supercomputers).

I should like to thank Philippe Binder, Jean-Pierre Boon, Dominique d'Humières, Tony Ladd, Alain Noullez and, in particular, Matthieu Ernst for stimulating discussions and encouragement.

\section{References}

[1] B. J. Alder and T. E. Wainwright, Phys.Rev.A1,18(1970).

[2] Y. Pomeau and P. Résibois, Phys.Rep.19,63(1975).

[3] I. de Schepper and M. H. Ernst, Physica 87A,35,(1977)

[4] D. Forster, D. R. Nelson and M. J. Stephen, Phys.Rev.A16,732(1977).

[5] R. B. Pandey, D. Stauffer, A. Margolina and J. G. Zabolitzky, J. Stat.Phys.34,427(1984).

[6] D. Frenkel, Phys.Lett.A121,385(1987)

[7] D. Frenkel and G. A. van Velzen, to be published.

[8] U. Frisch, B. Hasslacher and Y. Pomeau, Phys.Rev.Lett.56,1505(1986).

[9] D. d'Humières and P. Lallemand, Complex Systems 1,599(1987).

[10] J. Hardy, O. de Pazzis and Y. Pomeau, Phys.Rev.A13,1949(1976)

[11] U. Frisch, D. d'Humières, B. Hasslacher, P. Lallemand, Y. Pomeau and J.-P. Rivet, Complex Systems 1, 649(1987).

[12] J.-P. Boon and A. Noullez in Proceedings of workshop on 'Discrete kinetic theory, lattice gas dynamics and foundations of hydrodynamics', World Scientific, Singapore, 1989.

[13] P. M. Binder and D. d'Humières, Los Alamos preprint LA-UR-1341 (1988) and Proceedings of workshop on 'Discrete kinetic theory, lattice gas dynamics and foundations of hydrodynamics', World Scientific, Singapore, 1989.

[14] M. E. Colvin, A. J. C. Ladd and B. J. Alder, Phys.Rev.Lett.61,381(1988)

[15] G. Zanetti, preprint.

[16] R. F. Fox, Phys.Rev.A27,3216(1983).

[17] D. Frenkel and M. H. Ernst, submitted for publication. 